\title{
Multiphase sinusoidal oscillator using the CFOA pole
}

\author{
D.-S. Wu \\ S.-I. Liu \\ Y.-S. Hwang \\ Y.-P. Wu
}

Indexing terms: Multiphase sinusoidal oscillator, Amplifiers

Abstract: A multiphase sinusoidal oscillator using the parasitic poles of current feedback operational amplifiers (CFOAs) is presented. The oscillator can generate $n$ signals which are equal in amplitude and equally spaced in phase. The oscillator using the parasitic poles of CFOAs is suitable for high frequency applications and monolithic IC fabrication. The effects caused by the nonlinearity of the CFOA on the oscillation frequency and condition have been analysed. Experimental results show that a three-phase sinusoidal oscillator has a wide operating range from $10 \mathrm{kHz}$ to $10 \mathrm{MHz}$ and a large output voltage swing up to $7 \mathrm{~V}$ peak to peak while using $\pm 5 \mathrm{~V}$ power supply. The total harmonic distortion (THD) of each oscillation frequency is dominated by its third harmonic component which is at least $33 \mathrm{~dB}$ down from its fundamental.

\section{Introduction}

Multiphase sinusoidal oscillators (MSOs) play an important role in communications, signal processing and power electronics. A number of MSOs based on different techniques have been developed in literature [1-7]. MSOs implemented by the methods of References 1-4 exhibit good performance, but those circuits suffer complex circuitry from using a large number of components. On the other hand, both of the MSOs realised by active- $R$ technique [5] and operational transconductance amplifiers (OTAs) [6] feature simple circuit structure, but they are restrained by the limited operating range and output voltage swing.

Because CFOAs possess more extended bandwidths and higher slew rates than conventional operational amplifiers (OAs), they have been widely used in place of OAs as active devices in analog circuit applications [8, 9]. However, little attention has been paid on realisation of CFOA based MSOs.

In this paper, we propose a simple scheme for realising MSOs which features the following merits: using only the parasitic poles of CFOAs that makes it suitable for high frequency oscillation and monolithic IC fabrication, possessing large output voltage swing and moderately low

(C) IEE, 1995

Paper 1682G (E10), first received 17th March and in revised form 8th November 1994

The authors are with the Department of Electrical Engineering, National Taiwan University, Taipei, Taiwan, 10664, Republic of China

IEE Proc.-Circuits Devices Syst., Vol. 142, No. 1, February 1995
THD, and having simple circuit structure that only two resistors and one CFOA are required for each phase.

\section{Circuit description}

The circuit symbol and equivalent circuit for a CFOA including a compensation terminal are shown in Fig. 1

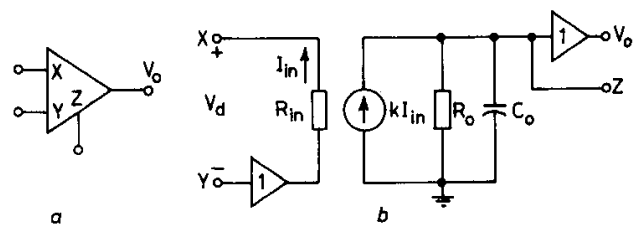

Fig. 1 CFOA

a Circuit symbol

b Equivalent circuit

[10]. The transfer function between the output and input terminals of the CFOA can be given by

$$
\frac{V_{0}}{V_{d}}=\frac{-k R_{0}}{R_{i n}\left(1+\frac{s}{\omega_{p 1}}\right)} \quad \omega_{p 1}=\frac{1}{R_{0} C_{0}}
$$

where $k$ is the current transfer ratio, $R_{\text {in }}$ is the input resistance of the inverting input terminal, $\left(R_{0} / / C_{0}\right)$ is the equivalent parasitic impedance at the compensation terminal, and $\omega_{p 1}$ is the CFOA dominant pole. According to eqn. 1, a CFOA can be used as a first-order low-pass filter.

Based on Fig. 1, the generalised circuit for realising an MSO is shown in Fig. 2. This oscillator is composed of

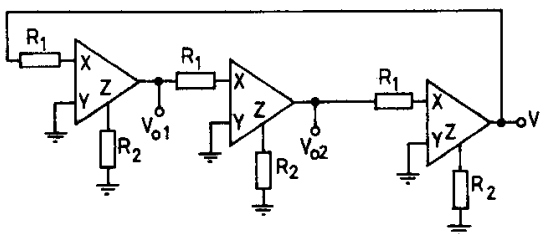

Fig. 2 Generalised circuit for realising multiphase oscillator

two resistors and one CFOA for each phase. Assuming that all CFOA in Fig. 2 are identical and $k=1$, the loop gain can be expressed as

$$
L(s)=\left(\frac{-G_{0}}{1+\frac{s}{\omega_{b}}}\right)^{n}
$$


Table 1: Frequency and condition of oscillation of multiphase oscillator

\begin{tabular}{lll}
$\begin{array}{l}\text { Number } \\
\text { of phase }\end{array}$ & $\begin{array}{l}\text { Frequency } \\
\text { of oscillation }\end{array}$ & $\begin{array}{l}\text { Condition of symmetrical } \\
\text { multiphase oscillation }\end{array}$ \\
\hline$n$ & $\omega_{0}$ & \\
3 & $\omega_{0}=1.732 \omega_{b}$ & $R_{b} \geqslant 2 R_{a}$ \\
5 & $\omega_{0}=0.727 \omega_{b}$ & $R_{b} \geqslant 1.236 R_{b}$ \\
7 & $\omega_{0}=0.482 \omega_{b}$ & $R_{b} \geqslant 1.110 R_{a}$ \\
$\vdots$ & $\vdots$ & $\vdots$ \\
$n$ & $\omega_{0}=\omega_{b} \tan \frac{\pi}{n}$ & $R_{b} \geqslant R_{b} \sec \frac{\pi}{n}$ \\
&
\end{tabular}

where

$$
\begin{aligned}
\omega_{b} & =\frac{1}{\left(R_{2} / / R_{0}\right) C_{0}}=\frac{1}{R_{b} C_{0}} \quad R_{b}=\left(R_{2} / / R_{0}\right) \\
G_{0} & =R_{b} / R_{a} \quad R_{a}=R_{1}+R_{i n}
\end{aligned}
$$

According to the Barkhausen criterion [11], the condition for the proposed circuit to provide sinusoidal oscillations of frequency $\omega_{0}$ is that

$$
\left(1+\frac{j \omega_{0}}{\omega_{b}}\right)^{n}-\left(-G_{0}\right)^{n}=0
$$

By expanding eqn. 5 , it is easy to show that eqn. 5 would have a solution only if the value of $n$ is odd $(n \geqslant 3)$. By equating the imaginary and real parts to zero, respectively, the frequency and condition of oscillation can be shown as

$$
\omega_{0}=\omega_{b} \tan \frac{\pi}{n}
$$

and

$$
R_{b} \geqslant R_{a} \sec \frac{\pi}{n}
$$

From eqns. 6 and 7, the oscillation frequency and condition of a three-phase sinusoidal oscillator $(n=3)$ can be expressed as

$$
\omega_{0}=\sqrt{ }(3) \omega_{b}
$$

and

$$
R_{b} \geqslant 2 R_{a}
$$

or

$$
R_{1} \leqslant \frac{R_{2} R_{0}}{2\left(R_{2}+R_{0}\right)}-R_{i n}
$$

According to eqns. 8 and 9 , this three-phase sinusoidal oscillator has the maximum and minimum oscillation frequencies when $R_{1}=0$ and $R_{1}=\left(R_{0} / 2\right)-R_{\text {in }}$, respectively.

Finally, the oscillation frequency and condition for realising an $n$-phase sinusoidal oscillator, equal in amplitude and equally spaced in phase, are summarised in Table 1 .

\section{Sensitivity analysis and frequency stability}

From the above analysis, the sensitivities of the passive and active components on oscillation frequency can be derived as

$$
\begin{aligned}
& S_{R_{a}}^{\omega_{0}}=S_{R_{b}}^{\omega_{0}}=S_{C_{0}}^{\omega_{0}}=-1 \\
& S_{R_{1}}^{\omega_{0}}=\frac{-R_{1}}{R_{1}+R_{i n}} \\
& S_{R_{i n}}^{\omega_{0}}=\frac{-R_{i n}}{R_{1}+R_{i n}} \\
& S_{R_{2}}^{\omega_{0}}=\frac{-R_{0}}{R_{2}+R_{0}} \\
& S_{R_{0}}^{\omega_{0}}=\frac{-R_{2}}{R_{2}+R_{0}}
\end{aligned}
$$

Note that eqns. 10 are valid for each $n$-phase oscillation circuit. All of the absolute values of the sensitivities are no more than one. The frequency stability factor $S_{F}$ is defined as Reference 12:

$$
S_{F}=\left.\frac{d \phi(u)}{d u}\right|_{\omega=1}
$$

where $u=\omega / \omega_{0}$ is the normalised frequency, and $\phi(u)$ represents the phase function of the open loop transfer function in Fig. 2. And the frequency stability factor $S_{F}$ can be derived as

$$
S_{F}=\frac{-n}{2} \sin \frac{2 \pi}{n}
$$

The value of $\left|S_{F}\right|$ is proportional to that of $n$, and is equal to 1.30 and $\pi$ while $n=3$ and $\infty$, respectively.

\section{Effects of the CFOA nonlinearity on oscillation} frequency and condition

Considering the effects of the CFOA nonlinearity, we can use a two-pole model to describe the performance of a nonideal CFOA. Thus, it is suitable to modify the current transfer ratio of a nonideal CFOA as

$$
k=\frac{1}{1+\frac{s}{\omega_{p 2}}}
$$

where $\omega_{p 2}$ is the CFOA second pole. Substituting eqn. 13 into eqn. 1 , we can carry out the characteristic equation of the three-phase oscillation circuit in Fig. 2 as

$$
a_{0}+a_{1} s+a_{2} s^{2}+a_{3} s^{3}+a_{4} s^{4}+a_{5} s^{5}+a_{6} s^{6}=0
$$

where

$$
\begin{aligned}
& a_{0}=1+\left(\frac{R_{b}}{R_{a}}\right)^{3} \\
& a_{1}=\frac{3}{\omega_{b}}+\frac{3}{\omega_{p 2}} \\
& a_{2}=\frac{3}{\omega_{b}^{2}}+\frac{9}{\omega_{b} \omega_{p 2}}+\frac{3}{\omega_{p 2}^{2}} \\
& a_{3}=\frac{1}{\omega_{b}^{3}}+\frac{9}{\omega_{b}^{2} \omega_{p 2}}+\frac{9}{\omega_{b} \omega_{p 2}^{2}}+\frac{1}{\omega_{p 2}^{3}} \\
& a_{4}=\frac{3}{\omega_{b}^{3} \omega_{p 2}}+\frac{9}{\omega_{b}^{2} \omega_{p 2}^{2}}+\frac{3}{\omega_{b} \omega_{p 2}^{3}} \\
& a_{5}=\frac{3}{\omega_{b}^{3} \omega_{p 2}^{2}}+\frac{3}{\omega_{b}^{2} \omega_{p 2}^{3}} \\
& a_{6}=\frac{1}{\omega_{b}^{3} \omega_{p 2}^{3}}
\end{aligned}
$$

IEE Proc.-Circuits Devices Syst., Vol. 142, No. 1, February 1995 
For simplicity, the fifth and the sixth order terms in eqn. 14 can be neglected because their coefficients are much smaller than that of the fourth and lower terms. Based on

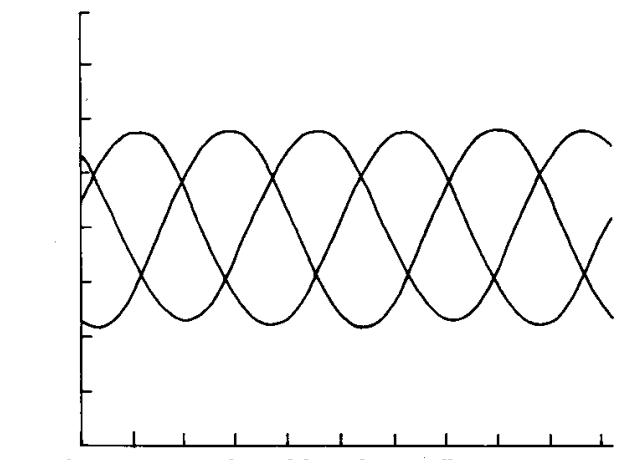

Fig. 3 Output waveform of three phase oscillator

$f_{0}=10.3 \mathrm{MHz}$

Horizontal scale is about $20 \mathrm{~ns} /$ div; vertical scale is $1 \mathrm{~V} /$ div
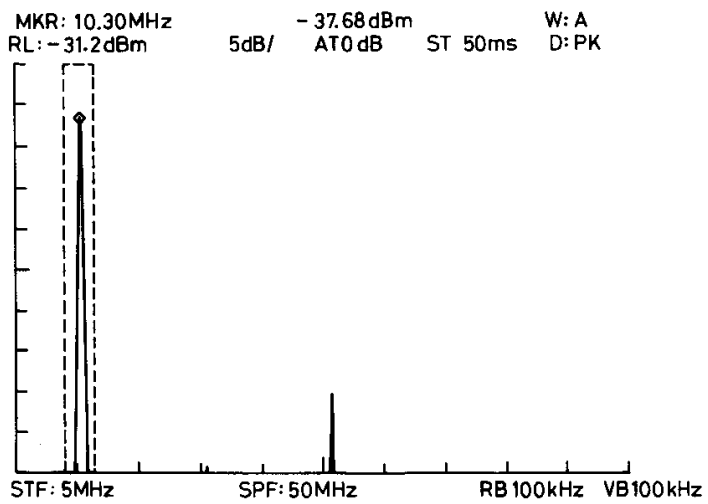

Fig. 4 Frequency spectrum of one output signal of Fig. 3

Horizontal scale is $5 \mathrm{MHz} / \mathrm{div}$; vertical scale is $5 \mathrm{~dB} / \mathrm{div}$

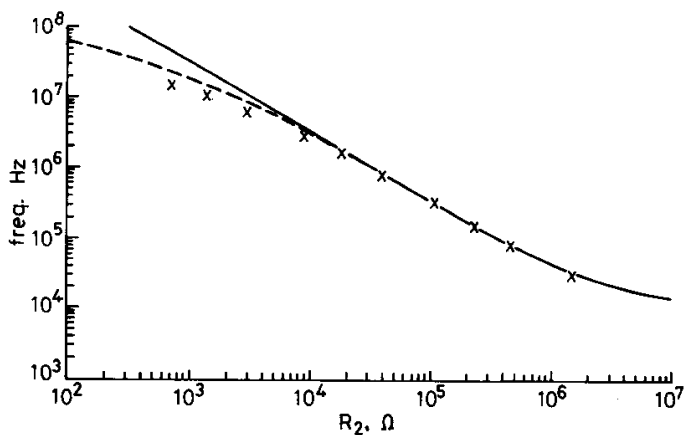

Fig. 5 Variation of oscillation frequency $f_{0}$ with resistor $R_{2}$

$-\ldots$ linear

$x \times x$ exp.

this condition, we would derive the approximate expressions of the oscillation frequency and condition as

$$
\omega_{0}=\lambda \omega_{b}
$$

and

$$
\boldsymbol{R}_{b} \geqslant \mu \boldsymbol{R}_{a}
$$

or

$$
R_{1} \leqslant \frac{R_{2} R_{0}}{\mu\left(R_{2}+R_{0}\right)}-R_{\text {in }}
$$

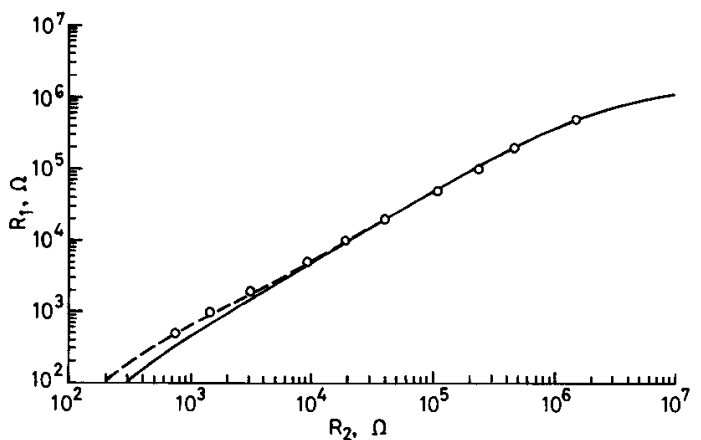

Fig. 6 Relationship of oscillation condition between $R_{1}$ and $R_{2}$

$\begin{array}{ll} & \text { linear } \\ \text { nonlin. }\end{array}$

OOO exp.

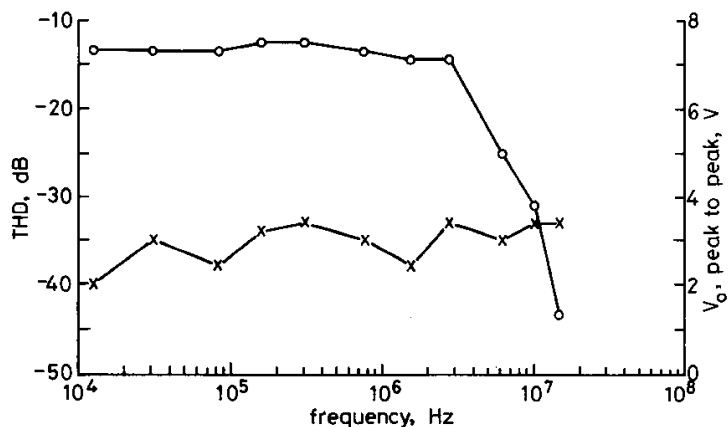

Fig. $7 T H D$ and output voltage swing of various oscillation frequencies

$\times \times \times$ THD

OOO $V_{0}$

where

$$
\begin{aligned}
& \lambda=\left(\frac{3}{1+8 \Omega+\Omega^{2}}\right)^{1 / 2} \\
& \mu=\left(\frac{9\left(1+8 \Omega+17 \Omega^{2}+8 \Omega^{3}+\Omega^{4}\right)}{\left(1+8 \Omega+\Omega^{2}\right)^{2}}-1\right)^{1 / 3} \\
& \Omega=\omega_{b} / \omega_{p 2}
\end{aligned}
$$

A detailed analysis indicates that the values of $\lambda$ and $\mu$ are equal to $\sqrt{(3)}$ and 2 , respectively, while $\Omega \rightarrow 0$. This consequence shows that the oscillation frequency and condition derived from the linear and nonlinear model of CFOA are almost equal when $\omega_{0} \ll \omega_{p 2}$.

\section{Experimental results and discussion}

To verify the theoretical analysis, the circuit of a threephase sinusoidal oscillator has been realised by a commercial CFOA (AD844AN). The typical values of $R_{i n}$ and $R_{0}$ of a CFOA are $50 \Omega$ and $3 \mathrm{M} \Omega$ [10], respectively. The total parasitic capacitance caused by the AD844AN and 
the circuit breadboard is about $8.3 \mathrm{pF}$ measured at each compensation terminal of the CFOAs. The second pole of the AD844AN is about $70 \mathrm{MHz}$ [13].

The output waveform of the three-phase sinusoidal oscillator at $10.3 \mathrm{MHz}$ is shown in Fig. 3, with $R_{1}=$ $1 \mathrm{k} \Omega, R_{2}=1.44 \mathrm{k} \Omega$, and $\pm 5 \mathrm{~V}$ power supply. Fig. 4 shows the frequency spectrum of one output signal of Fig. 3. It shows that the THD is dominated by the third harmonic component which is $33 \mathrm{~dB}$ down from the fundamental. The relationship between $R_{2}$ and $f_{0}\left(f_{0} \equiv\right.$ $\left.\omega_{0} / 2 \pi\right)$ is shown in Fig. 5 which indicates good agreement between the linear model prediction and experimental results while $f_{0}$ is under $1.5 \mathrm{MHz}$, but, for higher frequencies, the larger error between the linear model prediction and the measured results occurs. On the contrary, the nonlinear model prediction in high frequency range matches much better with the measured results than the linear model does.

The oscillation conditions between $R_{2}$ and $R_{1}$ are plotted in Fig. 6 which shows that the experimental results are well matched with the nonlinear model prediction. The THD and the output voltage swing of various oscillation frequencies are shown in Fig. 7. It shows that the output voltage swing remains almost constant when $f_{0}$ is under $3 \mathrm{MHz}$, and that the THD of each frequency is at least $33 \mathrm{~dB}$ down from its fundamental.

Again, we restudy both Fig. 5 and Fig. 7. It turns out that, due to the effects of the higher order poles and the slew rate; the output voltage swings decline rapidly; meanwhile, the measured oscillation frequencies deviate from the nonlinear model prediction when the oscillation frequency is above $3 \mathrm{MHz}$.

\section{Conclusions}

A simple scheme, which exploits the parasitic poles of the CFOAs, has been presented for realising an MSO. The three-phase sinusoidal oscillator has been built and tested. Experimental results which agree very well with the theoretical result are obtained. Because the frequency of oscillation (i.e. eqni. 6) is inversely proportional to the total parasitic capacitance at the compensation terminal of the CFOA, it means that a voltage controlled multiphase oscillator can be realised if each phase has a voltage-controlled grounded capacitor (VCGC) connected to the compensation terminal of the CFOA. A VCGC can be realised by the active- $R$ technique [14]. The capacitor [14] is a function of the gain bandwidth product (GBP) of the OAs. The GBP, however, is a biasing supply dependent parameter, so it is feasible to realise a VCGC by controlling the biasing supply of the circuit. The results are expected to be useful in analogue signal processing applications.

\section{References}

1 RAHMAN, A., and HAQUE, S.E.: 'A simple three-phase variablefrequency oscillator', Int. J. Electr., 1982, 53, pp. 83-89

2 MIKHAEL, W.B., and TU, S.: 'Continuous and switched-capacitor multiphase oscillators', IEEE Trans., 1984, CAS-31, pp. 280-293

3 RAMAMURTI, V.P., and RAMASWAMI, B.: 'A novel three-phase reference sinewave generator for PWM invertors', IEEE Trans., 1982, IE-29, pp. 235-240

4 KAPLAN, B.Z., and BACHAR, S.T.: 'A versatile voltage controlled three phase oscillator', IEEE Trans., 1979, IECI-26, pp. 192-195

5 ABUELMA'ATTI, M.T., and ALMANSOURY, W.A.: 'Active-R multiphase oscillators', IEE Proc. G, 1987, 134, pp. 292-293

6 IQBAL, A.K., MUSLIM, T.A., and NIGAR, M.: 'Tunable OTAbased multiphase sinusoidal oscillators', Int. J. Electr., 1992, 72, pp. 443-450

7 RABINOVICI, R., KAPLAN, B.Z., and YARDENI, D.: 'Fundamental topologies of three-phase $\mathrm{LC}$ resonators and their applications for oscillators', IEE Proc. G, 1987, 140, pp. 148-154

8 BOWERS, D.F. ALEXANDER, $M$, and BUXTON, J.: 'A comprehensive simulation macromodel for 'current feedack' operational amplifiers', IEE Proc. G, 1987, 137, pp. 137-145

9 WILSON, B.: 'Recent developments in current conveyors and current-mode circuits', IEE Proc. G, 1990, 137, pp. 63-77

10 Analogue devices AD844 data sheet

11 SEDRA, A.S., and SMITH, K.C.: 'Microelectronic circuits' (Holt, Rinehart and Winston, Inc., New York, 1987)

12 BHASKAR, DR, and SENANI, R. "New current-conveyor-based single-resistance-controlled/voltage-controlled oscillator employing grounded capacitors', Electron. Lett., 1993, 29, pp. 612-614

13 'Macro model of AD844AN in PSICE library' (MicroSim Corporation, California, 1992)

14 SIDDIQI, M.A.; and AHMED, M.T.: 'Realization of grounded capacitor with operational amplifiers and resistance', Electron. Lett., 1978,14 , pp. $633-634$ 